\title{
The causes of obesity: advances in molecular biology but stagnation on the genetic front
}

\author{
C.B ouchard \\ Physical A ctivity Sciences L aboratory, L aval U niversity, Ste-Foy, Q uébec, Canada
}

Exciting advances have been made in the field of obesity over the last few years particularly with respect to the molecular determinants of energy balance. A Ithough much remains to be uncovered, there is growing optimism that the causes of the susceptibility to be in positive energy balance will be identified. It is important, however, to appreciate the fact that the gains of the last few years have been almost exclusively made in the identification of molecules that play a role in the regulation of food intake, metabolic rate, substrate or nutrient partitioning, and adipose cell biology. This progress is absolutely critical for a better understanding of the determinants of energy balance and more will be learned in the years to come from the research fostered by these advances in molecular biology.

In contrast, little progress has been made during the same period with respect to the genetic basis of human obesity. If genes contribute to human obesity either by causing the disease (i. e. a necessary or a major gene effect) or by making people more susceptible to the development of an obese condition (i.e. susceptibility gene(s) of a polygenic system), they do so because of DNA sequence variation(s) affecting expression or function. U nfortunately, the spectacular gains in the understanding of the biology of energy balance of the last few years have not translated into significant advances on the genetic front. This is particularly striking when one realizes that there is not one obese human whose excess body mass and body fat can be explained by a specific mutation in one of the genes exerting its effects in relevant energy balance pathways, with the possible exception of the $M$ endelian syndromes which exhibit obesity as one of their characteristic features. But, we knew that

Corresponding author: C. Bouchard, Ph.D., Physical A ctivity Sciences Laboratory, Laval U niversity, Ste-Foy, Québec, G 1K 7P4 Canada

A bbreviations: QTL, Q uantitative trait loci. much before the cloning of the single-gene obesity mouse models.

\section{G enetic epidemiology}

H ow little we know about the true contribution of genetic variation to human obesity is illustrated by the current knowledge of the genetic epidemiology of the disorder [1]. H eritability estimates for B MI are quite heterogeneous. A ccording to twin studies, the heritability of B M I would be in the range of 40 to $70 \%$. The heritability estimates for $\mathrm{B} M \mathrm{I}$ derived from adoption studies tend to cluster around $30 \%$ or less. The heritability level is highest with twin studies, intermediate with nuclear family data, and lowest when derived from adoption data. When several types of relatives are used jointly in the same design, the heritability estimates typically cluster around 25 to $40 \%$ of the ageand gender-adjusted phenotype variance [1]. There is no clear evidence for a specific maternal or paternal effect and the common familial environmental effect is marginal. It is specially surprising that we still have no understanding of the true risk level for the development of an overweight or an obese state to which the first-degree relatives of an overweight or an obese person are exposed. We suspect that the risk level is significant and that it probably increases with the severity of the obese state of the affected relative but there is as yet no comprehensive and population-based data on these issues.

Several studies have dealt with complex segregation analysis of the B M I in panels of nuclear families. In general, they reported evidence for a multifactorial component and a major effect. However, in two reports, the major effect was non-M endelian. In a third paper, the major effect became $M$ endelian only when age and sex-related variations in the major effect were considered. In 5 of the 7 studies, the estimated gene frequency of the putative recessive gene ranged from about 0.2 to 0.3 [1]. H owever, since this 
putative gene has escaped detection up to now, it is impossible to verify whether this hypothesis is nonsense or is rooted in reality.

\section{M utations in rodent models}

E ven if no mutation has been found to be linked to a common form of human obesity, there is solid evidence for a role of specific mutations in the causation or in the increase risk of obesity in mouse and rat. To date, about 20 such mutations have been identified or reported to exist as evidenced by the single-gene locus models or by the quantitative trait loci (QTL) revealed by crossbreeding experiments with informative inbred strains [2]. It is humbling to be forced to recognize that none of these single-gene mutations and none of the genetic polymorphisms responsible for the QTL effects have been uncovered in humans. A s a matter of fact, none of the mutations responsible for the QTL effects have been identified in the experimental animal models. This alone represents unequivocal support for the view expressed herein that while important advances have been registered in the biochemical and molecular biology domain, the genetic aspect of the disease is stagnant.

\section{A ssociation and linkage studies}

A good number of association and linkage studies with obesity phenotypes have been reported and the results have been recently reviewed [3]. B riefly, most association studies between candidate genes and $\mathrm{BMI}$ or indicators of body fat have been negative. Those that have reported evidence for a significant association were based on small sample sizes and the evidence was generally weak. The reports with no evidence of association with markers of candidate genes suffer from the same limitations. With very few exceptions, none of the studies have been replicated.

The linkage studies based on mutations in candidate gene loci, microsatellites or other markers have also been disappointing. Four markers have been found to be linked to BMI or body fat phenotypes with slightly better than suggestive evidence levels. They are A CP 1 (2p25), a marker in the vicinity of tumour necrosis factor- $\alpha(6 p 21.3)$, the K ell blood group (7q33) and the A DA gene (20q13.1) [3]. H undreds of markers based on the sib-pair method have been negative. No replication study has been published so far; however, two recent papers have independently reported weak evidence for linkage between markers flanking the human ob gene on 7q31.1 and severe obesity $[4,5]$. The latter observations are concordant with the finding of a linkage between the K ell blood group and body fat phenotypes in the Q uébec Family Study [6].
$\mathrm{N}$ ew insights on obesity genes

In this issue of D iabetologia, D rs. G uerre-M ilo et al. discuss the role that selected genes may play in the aetiology of obesity and adipose cell differentiation. The contributions of the agouti, ob, $\mathrm{db}$, and fat genes to rodent energy balance and obesity are appropriately reviewed. They then focus on the potential role of selected transcription factors and DNA -binding proteins in the differentiation of adipose cells. H ence, peroxisome proliferator activated receptor gamma 2 (PPA R $\gamma 2$ ), C/E BP transcription factors $(\alpha, \beta$ and $\delta$ ) and HMGI-C DNA binding protein are proposed as potential candidate genes for obesity based on the hypothesis that these molecules are involved in adipogenesis.

Of course, this list of putative genes can be expanded considerably. A s a matter of fact, all the molecules involved in the determination of energy balance, nutrient partitioning and adipose tissue expansion could legitimately be in that listing. The merit of the article by Guerre-M ilo and collaborators is to identify and justify molecules that have received only limited attention by those interested in the genetics of obesity. H owever, while the paper is a fine contribution to the molecular biology of adipogenesis, it does not provide us with new insights into the genetics of obesity. The latter will be achieved only if mutations in the genes encoding these molecules or in their regulatory sequences are found and shown to be associated or linked with obesity phenotypes or adipose tissue expansion.

It is obvious that these genes and a score of others deserve to be included in the list of candidate genes to be tested in the family studies of obesity currently in progress. Perhaps they will eventually move from being considered as molecular determinants of adipogenesis to obesity-causing genes; but, for the moment, no mutations with functional implications have been uncovered in these genes.

\section{References}

1. Bouchard C (1994) Genetics of obesity: overview and research directions. In: B ouchard C (ed) The genetics of obesity. CR C Press, B oca R aton, FL, pp 223-233

2. Chagnon $Y, B$ ouchard $C$. The genetics of obesity: advances from rodent studies. Trends in G enetics (in press)

3. B ouchard C, Pérusse L (1996) Current status of the human obesity gene map. O besity R esearch 4: 81-90

4. R eed DR, D ing Y, X u W, Cather C, Green ED, Price RA (1996) Extreme obesity may be linked to markers flanking the human O B gene. D iabetes 45: 691-694

5. Clement K, Garner C, H ager J, Philippi A, L eD uc C, Carey $A$ et al. (1996) Indication for linkage of the human OB gene region with extreme obesity. D iabetes 45: 687-690

6. B orecki IB, Rice T, Pérusse L, B ouchard C, R ao D C (1994) A $n$ exploratory investigation of genetic linkage with body composition and fatness phenotypes: the Québec Family Study. $O$ besity R esearch 2: 213-219 